\title{
Claude Dreux (1929-2021)
}

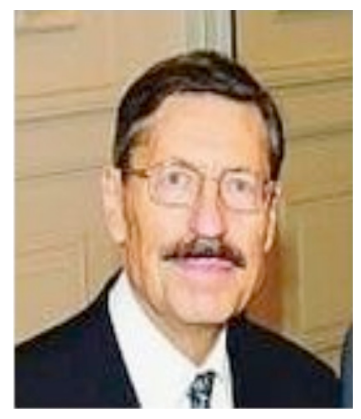

À la suite du décès du Professeur Claude Dreux le 16 mars 2021, c'est à moi que revient aujourd'hui le triste privilège de rédiger cette notice. Il a été et restera toujours pour moi «le patron». Je suis entré dans son service en avril 1970 en tant qu'interne et y suis resté jusqu'à sa retraite hospitalière en 1994. Claude Dreux a été l'un des plus jeunes qui ait réussi le difficile concours du Pharmacopat. Comme pharmacien-chef il dirigeait donc, lors de mon arrivée, conjointement la pharmacie et le service de biochimie qui effectuait également les examens courants d'hémostase, à l'hôpital Saint-Louis, temple de l'hématologie avec le très important service du Professeur Jean Bernard. L'hémostase a d'ailleurs été ma première «paillasse» d'interne, ce qui était prémonitoire puisqu'on sait à présent que les plaquettes sanguines contiennent la quasi-totalité de la sérotonine sanguine circulante. Avant la fin de mon internat, «le patron» m'a demandé de poursuivre ses travaux de thèse de sciences qui portaient sur la fluorimétrie et le dosage de composés indoliques (métabolites du tryptophane) dont la sérotonine, molécule fascinante que Claude Dreux a chérie toute sa vie. C'est ainsi que je cosignais avec lui en 1973 mon premier article scientifique portant sur la détermination de la sérotonine plaquettaire, par une méthode dérivant de la technique initiale de Bogdanski, Pletscher et Brodie utilisée pour le cerveau. Cela illustre un aspect très positif de la personnalité de Claude Dreux: quand il vous accordait sa confiance, il vous «poussait » autant qu'il le pouvait et mettait à votre disposition ses connaissances, son expérience et les moyens nécessaires à la recherche. En 1975, il a ainsi facilité mon premier séjour à Bâle auprès des Professeurs Pletscher et Da Prada. «Le patron» m'a ensuite permis de remplacer pour le dosage de la sérotonine la fluorimétrie par la radioenzymologie (technique mise au point par le groupe de J. Axelrod), puis par la chromatographie liquide qu'il appliquait avec d'autres collègues (B. Bousquet, J.P. Garnier) aux dosages du tryptophane «libre » et lié à l'albumine ainsi que d'autres dérivés de cet acide aminé essentiel (kynurénines, indoles..., si importants pour les interactions hôte/microbiote). Ceci a permis de «démocratiser» les dosages des hydroxyindoles et de réussir la première transplantation d'une tumeur carcinoïde humaine à la souris (Ganem et al., 1991) et cela reflète une seconde caractéristique de Claude Dreux: c'était un analyste toujours à l'affût de nouvelles techniques, de nouvelles applications ou sujets d'étude.

À la fin des années 1970, lors de la séparation biochimie/pharmacie, «le patron » a choisi le laboratoire. Son côté analyste et son appétence pour tout ce qui était nouveau lui ont permis de faire de son service un lieu d'innovations permanentes dans des domaines très variés : - l'automatisation et l'informatisation du service dès le début des années 1970 (F. Tabuteau);

- l'introduction de la chromatographie en phase gazeuse pour l'analyse des stéroïdes à la même période (Chevins et al., 1974). Cette analyse des stéroïdes a été considérablement développée par Jean Fiet via la radioimmunologie avec production locale d'anticorps polyclonaux (ce que nous appelions entre nous la «paillasse» lapins), puis monoclonaux, à tel point que «le patron» a accepté d'autonomiser ce secteur hormonologie en service lors du transfert dans le nouvel hôpital Saint-Louis ;

- l'acquisition au milieu des années 1980 d'un spectromètre de masse initialement dévolu à la pharmacologie des anticancéreux (B. Gourmel);

- la création au tout début des années 1990 d'un secteur de génétique moléculaire avec l'aide de M. Vidaud et J. L. Laplanche, centré sur l'étude des maladies à prions.

Ceci nous amène aux deux principales thématiques défendues par Claude Dreux au cours de sa carrière. La première résulte de ses contacts avec les Professeurs Pletscher et Da Prada qui ont été les premiers à proposer la plaquette sanguine comme «modèle » de neurone. C'est à partir de ce concept qu'il avait adopté et qui a permis de nombreuses avancées en psychiatrie que Claude Dreux a créé avec les Professeurs Deniker, Loo et Zarifian la Psychiatrie Biologique française (Deniker et al., 1980; Giret et al., 1980). La seconde thématique chère au «patron» était encore plus visionnaire: bien avant les 
crises de l'hormone de croissance contaminée et de la «vache folle» (années 1990), il a mesuré toute l'importance pour la santé publique de l'infectiosité de la protéine prion lorsqu'elle est mal repliée, un concept qui remet en cause les fondements même de la biologie puisque, selon le dogme, le caractère infectieux ne peut être porté que par un acide nucléique. Nos premiers résultats sur le sujet ont d'ailleurs été présentés devant la Société de Biologie (Chatelain et al., 1984). En outre, «le patron» a accueilli dans son service Jacqueline Chatelain, une collaboratrice de Françoise Cathala dont l'unité INSERM consacrée aux agents transmissibles non conventionnels n'avait pas été maintenue lors de son départ en retraite car « cette recherche était marginale et ne concernait pas la santé publique». Par la suite, notre service est devenu référent national auprès de l'Institut de Veille Sanitaire pour la maladie de CreutzfeldtJakob. Enfin, Claude Dreux a eu le grand plaisir de faire docteur honoris causa de l'université Paris V le professeur Stanley Prusiner en 1996, un an avant son prix Nobel de Médecine obtenu pour la découverte des prions (PROteinaceous INfectious particle ou particule protéique infectieuse), terme qu'il a proposé dès 1982 .

Cet hommage ne serait pas complet si on ne rappelait pas les nombreuses fonctions du «patron» en dehors de son service hospitalier : professeur de Biochimie à la faculté des Sciences Pharmaceutiques et Biologiques puis Doyen de cette même faculté; vice-président de l'université Paris V; directeur du Comité d'éducation sanitaire et sociale de la pharmacie française (Cespharm) pendant de nombreuses années après sa retraite universitaire. Claude Dreux était membre des Académies Nationales de Pharmacie (qu'il a présidée en 1997) et de Médecine ainsi que de l'Académie Royale de Médecine de Belgique. Il a également été Président de la Société de Biologie de 2000 à 2004, puis vice-président de 2004 à 2008.

Toutes ses activités au plus haut niveau lui ont valu la reconnaissance des autorités de tutelle avec l'attribution des grades d'Officier dans l'Ordre de la Légion d'Honneur et de Commandeur dans l'Ordre National du Mérite.
Homme de conviction, Claude Dreux était très attaché à la profession de pharmacien dont il connaissait tous les aspects. Formé à Lariboisière dans le service de Maurice Louis Girard, il a maintenu et développé la biologie pharmaceutique avec ses collègues Alain Lemonnier, Jean Agneray et François Rousselet et préparé l'avènement du CHU pharmaceutique. Il était persuadé de l'importance des relations pharmaciensbiologistes/cliniciens et affirmait que, comme en recherche, l'interprétation des résultats d'analyse était aussi importante que l'analyse elle-même, pourvu que celle-ci soit fiable.

Merci, Cher Professeur Dreux, pour tout ce que vous avez apporté à toutes celles et ceux qui ont eu la chance de vous côtoyer. Nous ne vous oublierons jamais. Reposez en paix.

Jean-Marie Launay

\section{Références}

Chatelain, J., Haimart, M., Launay, J.M., Baille, V., Dreux, C., Cathala, F. (1984). Sérotoninémie et histaminémie chez des ovins endémiquement atteints de tremblante (scrapie) : premiers résultats. C R Soc Biol, 178, 664-670.

Chevins, R., Launay, J.M., Julien, R., Dreux, C. (1974). Analysis of steroid hormones by gas-liquid chromatography. 17ketosteroids, pregnanediol and pregnanetriol in the urine. Clin Chim Acta, 55, 333-343.

Deniker, P., Loo, H., Zarifian, E., Cuche, H., Benyacoub, K., Escande, C., Bousquet, B., Dreux, C. (1980). Measurement of the plasmatic level of free and protein-bound tryptophan in mental pathology. Neuropsychobiology, 6, 132-139.

Ganem, Y.J., Launay, J.M., Debons-Guillemin, M.C., Lasneret, J., Roucayrol, A.M., Lesser, J., Peries, G., Dreux, C. (1991). First heterotransplantation of a human carcinoid tumor into nude mice. Cancer, 68, 893-902.

Giret, M., Launay, J.M., Dreux, C., Zarifian, E., Benyacoub, K., Loo, H. (1980). Modifications of biochemical parameters in blood platelets of schizophrenic and depressive patients. Neuropsychobiology, 6, 290-296. 